\title{
Delivery of a functional anti-trypanosome Nanobody in different tsetse fly tissues via a bacterial symbiont, Sodalis glossinidius
}

\author{
Linda De Vooght ${ }^{1 *}$, Guy Caljon ${ }^{1,2,3}$, Karin De Ridder $^{1}$ and Jan Van Den Abbeele 1,4 $^{*}$
}

\begin{abstract}
Background: Sodalis glossinidius, a vertically transmitted microbial symbiont of the tsetse fly, is currently considered as a potential delivery system for anti-trypanosomal components that reduce or eliminate the capability of the tsetse fly host to transmit parasitic trypanosomes, an approach also known as paratransgenesis. An essential step in developing paratransgenic tsetse is the stable colonization of adult flies and their progeny with recombinant Sodalis bacteria, expressing trypanocidal effector molecules in tissues where the parasite resides.

Results: In this study, Sodalis was tested for its ability to deliver functional anti-trypanosome nanobodies (Nbs) in Glossina morsitans morsitans. We characterized the in vitro and in vivo stability of recombinant Sodalis (recSodalis) expressing a potent trypanolytic nanobody, i.e. Nb_An46. We show that recSodalis is competitive with WT Sodalis in in vivo conditions and that tsetse flies transiently cleared of their endogenous WT Sodalis population can be successfully repopulated with recSodalis at high densities. In addition, vertical transmission to the offspring was observed. Finally, we demonstrated that recSodalis expressed significant levels (ng range) of functional Nb_An46 in different tsetse fly tissues, including the midgut where an important developmental stage of the trypanosome parasite occurs.
\end{abstract}

Conclusions: We demonstrated the proof-of-concept that the Sodalis symbiont can be genetically engineered to express and release significant amounts of functional anti-trypanosome Nbs in different tissues of the tsetse fly. The application of this innovative concept of using pathogen-targeting nanobodies delivered by insect symbiotic bacteria could be extended to other vector-pathogen systems.

Keywords: (3-10), Sodalis glossinidius, Symbiont, Paratransgenesis, Recombinant, Glossina, Delivery, Functional, Nanobody, In vivo, Midgut

\section{Background}

The contemporary response to vector-borne infectious diseases still mainly relies on low-technology interventions, with a major emphasis on vector control through the use of insecticides. As vector-borne diseases continue to present significant threats to human, animal and plant health, there is an urgent need to develop control efforts that remain effective over time. Genetically modified disease vectors that are rendered resistant (refractory) to pathogen transmission can provide unique tools for developing new or complementing existing control strategies (reviewed by [1]). Paratransgenesis is one

\footnotetext{
* Correspondence: Idevooght@itg.be; jvdabbeele@itg.be

'Department of Biomedical Sciences, Unit of Veterinary Protozoology,

Institute of Tropical Medicine Antwerp (ITM), Antwerp, Belgium

Full list of author information is available at the end of the article
}

such approach that aims to reduce vector competence by genetically modifying symbionts of disease vectors and has been demonstrated for Rhodnius prolixus, the triatomine vector of Chagas disease [2] and Anopheles gambiae [3,4], the principal malaria vector in Africa.

A paratransgenic approach in tsetse flies, the sole vector of major African trypanosome parasites (T. brucei sp. and T. congolense), is of high interest since tsetse flies are not amenable to germ-line transformation due to their viviparous reproductive biology (intrauterine development and parturition of live offspring [5]). The tsetse fly harbors a natural commensal bacterium i.e. Sodalis glossinidius, which is ideally suited as a paratransgenic platform organism since it i) resides in different tsetse tissues that are in close proximity to pathogenic trypanosomes (e.g. midgut) [6]; ii) can be cultured and genetically modified in vitro 
$[7,8]$; iii) is maternally transmitted to the offspring [9] and iv) is restricted to the tsetse host niche ensuring that this symbiont is a safe candidate for use in the paratransgenic strategy [10].

The characteristics of the selected effector molecule will largely determine the efficacy and specificity of this paratransgenesis approach. Low specificity will generally result in bystander effects that could have a direct or indirect fitness cost for the arthropod. The use of highly specific compounds from the adaptive immune system of vertebrates such as antibody derived fragments is likely to enable highly specific effects without conferring a selective disadvantage to the (para)transgenic arthropods [11]. Nanobodies ${ }^{\oplus}$ (Nbs), representing the smallest known intact antigen-binding fragments derived from camelid heavy-chain only antibodies (HCAbs) [12], are therefore considered as excellent candidates to increase the immune competence of tsetse. Nanobodies targeting distinct epitopes of the variant-specific surface glycoprotein (VSG), abundantly present on the surface of bloodstream trypanosomes have already been identified, some of which were shown to exert direct in vitro and in vivo trypanolytic activity by interfering with the complex endocytotic machinery that is organized in the flagellar pocket of this parasite [13]. Recently, we developed recombinant Sodalis (recSodalis) strains expressing functional anti-trypanosome nanobodies. These strains were shown to release considerable amounts of functional anti-trypanosome $\mathrm{Nbs}$ to the extracellular culture environment and to have no negative effects on the bacterium in an in vitro context [14].

Another prerequisite in developing paratransgenic tsetse flies is the development of a methodology that allows the stable repopulation of tsetse flies with recSodalis strains expressing trypanosome-interfering proteins in insect tissues where trypanosome parasites reside. Previously, recSodalis expressing green fluorescent protein (GFP) has been successfully introduced into tsetse through thoracic microinjection [6]. Here, GFP-expressing Sodalis was found to be present in the haemolymph and gut tissues of injected females and their progeny. However, to date no studies have focused on the densities whereby genetically modified Sodalis, expressing heterologous genes, are maintained within the fly and the efficiency of their transmission to the offspring.

In this study, a recSodalis strain was tested for its ability to deliver functional anti-trypanosome $\mathrm{Nbs}$ in the tsetse fly Glossina morsitans morsitans. The in vivo long-term stability of the recombinant strain and transmission to the progeny was measured using a quantitative PCR (qPCR) analysis. We show that recSodalis expressing a potent trypanolytic nanobody, i.e. Nb_An46, was stably maintained in vivo only when the WT Sodalis population was significantly reduced ( $>95 \%$ of the normal population) prior to
recSodalis introduction. Furthermore, we demonstrated that significant levels of functional Nb_An46 accumulated in different fly tissues, including the midgut where an important developmental stage of the trypanosome parasite occurs.

\section{Results}

\section{In vitro culture characteristics of recombinant Sodalis expressing Nb_An46}

Prior to the introduction of recSodalis into experimental flies, the $\mathrm{Nb}$ expression profile, in vitro growth rate and plasmid stability of recSodalis expressing a FliCpelBNb_An46 fusion protein (Sod:FliCpelBNb46fliC) was established. Extracellular Nb_An46 expression was confirmed by Western blot analysis of supernatant from cultures grown to the beginning of stationary phase $\left(\mathrm{OD}_{600} 0.5\right.$ 0.6) (Figure 1). Nb_An46 expression and release was quantified at different time points during bacterial growth over a 10-day period by measuring the concentration of active $\mathrm{Nb}$ in the whole cell lysate and culture medium using a VSG-binding ELISA assay. Functional $\mathrm{Nb}$ _An 46 was expressed from day 2 onwards and accumulated in the culture medium to a concentration of $88 \mathrm{ng} / \mathrm{ml}$ by day 10 (Figure 2B). RecSodalis showed normal growth kinetics (Figure 2A) with cell population doubling times comparable to a WT Sodalis strain i.e., $15.0 \mathrm{hrs}$ and $14.8 \mathrm{hrs}$ respectively. The number of plasmid copies per cell was estimated to be approximately 20 during the lag and exponential phases of Sod: FliCpelBNb46fliC grown in the presence of antibiotic selection. The stability of the FliCpelBNb46fliC plasmid in recSodalis was measured by maintaining the recombinant bacteria in log phase growth for 27 generations in liquid MM medium in the absence of antibiotic selection. Colony counts on antibiotic-selective plates showed that $94 \%$ of the Sodalis population remained antibiotic resistant after 27 generations (corresponding to a 2 -month test period) (Table 1 ).

\section{Prior reduction of WT Sodalis is a prerequisite for an efficient host colonization with recSodalis}

We explored the capability of recSodalis to colonize the tsetse fly after introduction through intrathoracic injection. We first evaluated the necessity of the prior reduction of the WT Sodalis population in tsetse for recSodalis to establish and colonize its host. In female flies that received 3 streptozotocin supplemented blood meals, the WT Sodalis population was reduced by $95 \%$ and $88 \%$ in abdomen and thorax tissues respectively, compared to flies fed on normal blood. This treatment did not affect the obligatory Wigglesworthia symbiont population that mainly resides in the tsetse fly abdomen (Additional file 1: Figure S1). Next, the in vivo persistence and growth of the recombinant bacteria in streptozotocin- 


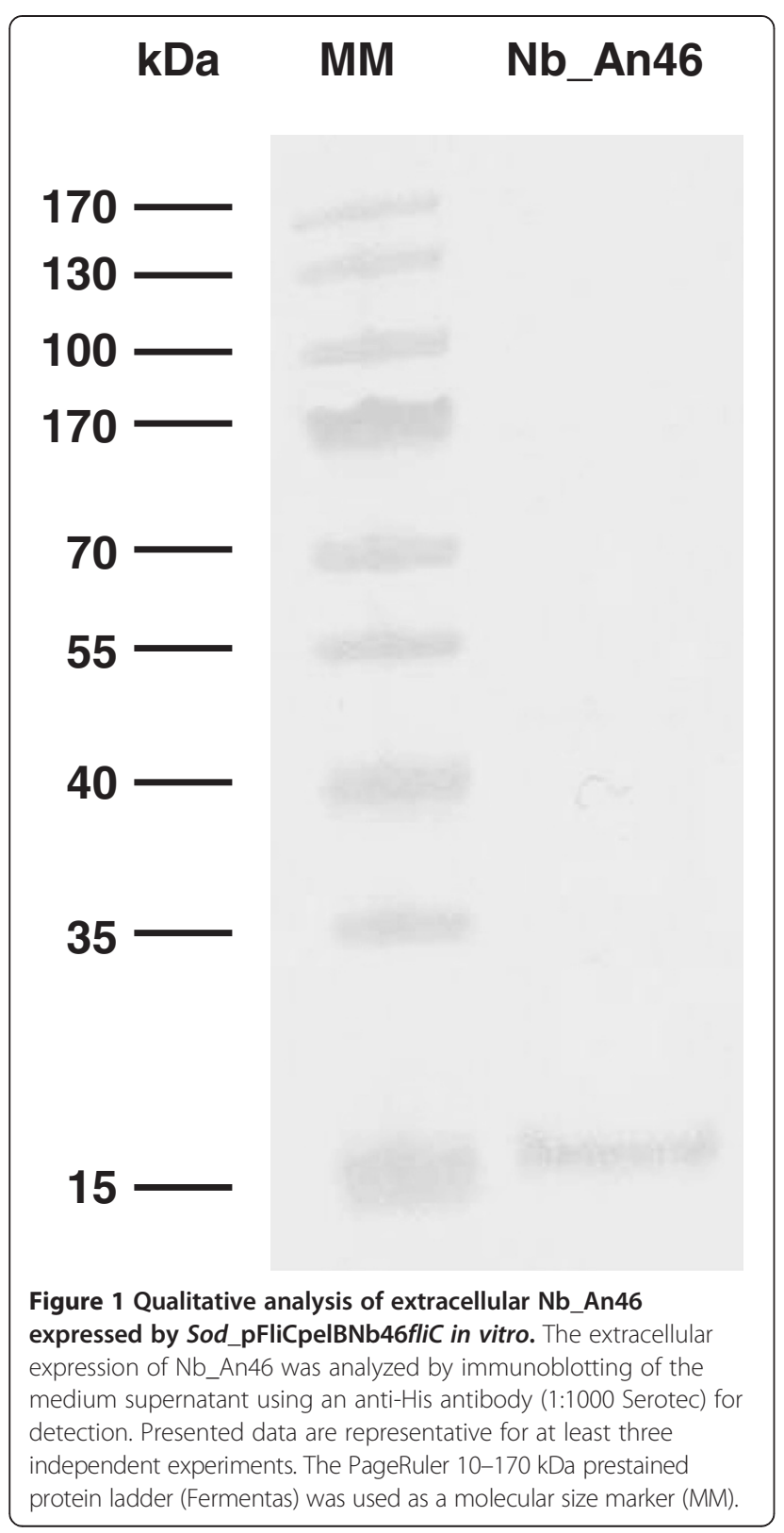

treated and non-treated flies injected intrathoracically with $5 \times 10^{4}$ CFU Sod_FliCpelBNb46fliC was evaluated using qPCR (Figure 3). In streptozotocin-treated flies, recSodalis was able to proliferate inside its host, reaching densities of 10 - and 20 -fold the initial injected dose in respectively abdomen and thorax, whereas in non-treated flies the injected recSodalis population was not able to expand and remained present at its initial density.

\section{The effect of differential doses of recSodalis inoculum on} tsetse fly colonization and viability

We determined the optimal dose for recSodalis injection in terms of host colonization and its viability. For this, streptozotocin-treated male flies were microinjected with either $5 \times 10^{4}, 5 \times 10^{5}, 10^{7}$ and $5 \times 10^{7} \mathrm{CFU}$ Sod_FliCpelBNb46fliC and recSodalis densities in abdomen and thorax tissues were determined 7 and 14 days postinjection (dpi) using qPCR (Figure 4). Injection of $5 \times$ $10^{7} \mathrm{CFU}$ resulted in an increased fly mortality (up to $59 \%$ mortality $14 \mathrm{dpi}$ ), whereas limited mortality $(\leq 25 \%$ $14 \mathrm{dpi}$ ) was observed within the other injection groups. In these groups, recSodalis was able to repopulate the abdomen and thorax to densities comparable to natural Sodalis levels present in WT flies (on average respectively $2.5 \times 10^{6}$ and $1.5 \times 10^{6} \mathrm{CFU}$ ), demonstrating that a wide range of recSodalis doses (i.e. $5 \times 10^{4}$ to $1 \times 10^{7}$ ) are suitable to initiate colonization without affecting fly viability.

recSodalis persists in the tsetse fly and is vertical transmitted to the offspring but at a low extent

The in vivo persistence of Sod_FliCpelBNb46fliC was evaluated by qPCR based estimation of the amount of recSodalis CFU in abdomen and thorax tissues of streptozotocin-treated male flies injected with $1 \times 10^{7}$ recombinant CFU over a 28 day period (Figure $5 \mathrm{~A}$ ). Sod_FliCpelBNb46fliC was able to remain present at high densities in abdomen and thorax tissues of experimental flies throughout the course of the 28-day observation period. In flies injected with Sod_FliCpelBNb46fliC, the entire Sodalis population in abdomen and thorax remained recombinant. Next, we evaluated recSodalis densities in the haemolymph and midgut tissues of flies injected with $1 \times 10^{7}$ recombinant CFU (Figure 5B). Sod_FliCpelBNb46fliC was able to reach the fly midgut where it persisted at densities between $5 \times 10^{4}$ and $1 \times 10^{5} \mathrm{CFU}$ (DNA equivalent) throughout the 21-day observation period. In these flies the obligatory Wigglesworthia symbiont population was not affected by the presence of Sod_FliCpelBNb46fliC (Additional file 1: Figure S2) nor did we observe any effect upon the fecundity of female recSodalis colonized flies. Transmission dynamics of the recombinant bacteria to the $F_{1}$ progeny was evaluated by qPCR. Sod_FliCpelBNb46fliC was transmitted to the $F_{1}$ generation, although nonplasmid containing Sodalis were dominant in these flies. Indeed, Sod_FliCpelBNb46fliC constituted only 7 and $5 \%$ of the entire Sodalis population in respectively abdomen and thorax (Figure 6).

\section{Functional Nb_An46 is expressed in different tsetse fly tissues}

$\mathrm{Nb}$ _An46 expression in flies injected with $1 \times 10^{7}$ Sod_FliCpelBNb46fliC CFU was quantified using a VSG-binding ELISA. Nanobody concentrations were determined at different time points post-injection in whole abdomen and thorax extracts, haemolymph and midgut (Figure 7). Functional Nb_An46 accumulated in haemolymph and 


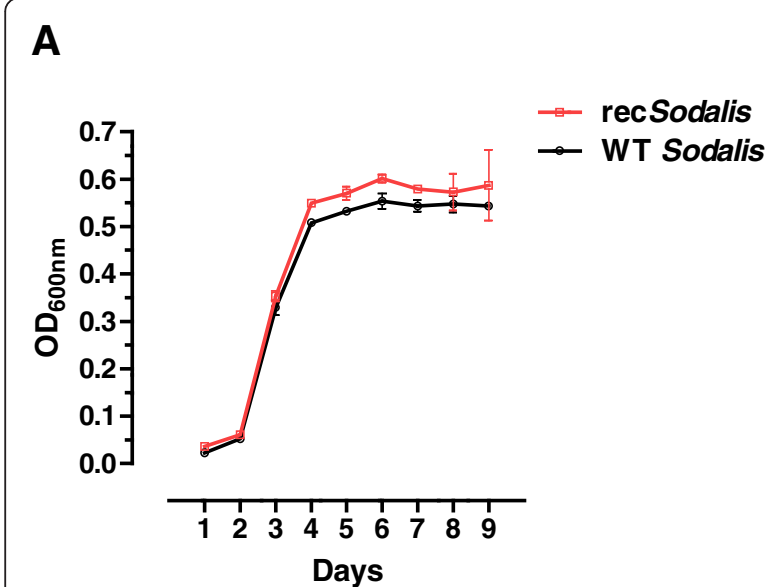

B

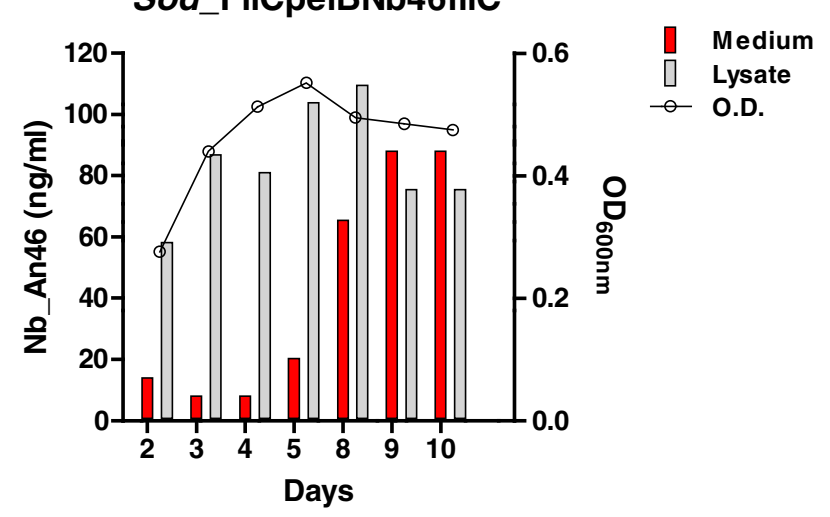

Figure 2 In vitro characteristics of recSodalis expressing Nb_An46. A) Growth curve analysis of WT Sodalis and Sod_pFliCpelBNb46fliC. The error bars show the \pm SD of two biological replicates. Samples were taken every 24 h. B) ELISA-based Nb_An46 quantitation (bar-chart) of the intra- and extracellular nanobody concentration produced by Sod_pFliCpelBNb46fliC at selected time points in relation to bacterial cell density $\left(\mathrm{OD}_{600 \mathrm{~nm}}\right.$, solid line) using a $6 \times$ His tag specific detection antibody. Values are presented as ng recombinant protein per ml culture medium.

thorax samples of injected flies over time, indicating a continuous transgene expression by recSodalis in these tissues. In the thorax the Nb_An46 concentration increased from $22 \mathrm{ng}$ on day 14 post-injection to $35 \mathrm{ng}$ on day 21. Although significant quantities of active Nb_An46 were detected in abdomen and midgut using a VSGbinding ELISA, the accuracy of this quantitation could have been hampered by the abundant presence of proteolytic enzymes in the tsetse fly gut, probably resulting in fast degradation of the nanobodies in the tissue homogenates and possibly resulting in the underestimation of the active $\mathrm{Nb}$ content of the non-digestive part of the tsetse fly gut.

\section{Discussion}

The aim of this study was to develop a functional Sodalisbased shuttle system that is able to deliver and express anti-trypanosome nanobodies within the tsetse fly. In this study a recombinant Sodalis strain was used expressing a potent trypanolytic nanobody Nb_An46 through a plasmid-based expression system. The functionality of the system was assessed by monitoring if recSodalis bacteria can (1) establish a stable population in the tsetse fly over time, (2) express significant levels of active

Table 1 Stability of pFliCpelBNb46FliC in Sodalis under nonselective growth conditions

\begin{tabular}{ll}
\hline Generations $(\mathbf{n})$ & Plasmid-containing CFU (\%) \\
\hline 10 & $\mathbf{1 0 0}$ \\
15 & $\mathbf{7 7}$ \\
20 & $\mathbf{9 1}$ \\
27 & $\mathbf{9 4}$
\end{tabular}

Plasmid stability was expressed as the ratio between the number of colonies formed on MM blood agar plates with $(50 \mu \mathrm{g} / \mathrm{ml})$ and without kanamycin. anti-trypanosome nanobodies in the tsetse fly and (3) be efficiently transferred to the offspring.

Although the growth characteristics of $\mathrm{Nb}$-expressing recSodalis were shown to be similar with those of the WT Sodalis in in vitro culture conditions it is plausible that in the tsetse fly in vivo environment the endogenous WT bacteria could have a competitive advantage over the introduced recombinant bacteria. Our results clearly suggest that recSodalis is competitive with WT Sodalis in in vivo conditions, however the maximal total Sodalis density in the inner tsetse fly environment seems to be limited to approximately $5 \times 10^{6} \mathrm{CFU}$. Indeed, only when the existing WT Sodalis population is priorly reduced $(>95 \%)$ by a selective antibiotic treatment, the introduced recSodalis population is able to maintain and proliferate to comparable density levels observed for wildtype Sodalis in normal tsetse flies. This recSodalis population is then able to outgrow the WT population which remains present at low density. In contrast, in non-treated flies recSodalis is not able to displace the WT Sodalis population but remains present at low density, confirming the importance of creating a WT Sodalis deprived niche that allows recSodalis to proliferate inside its host.

An important factor when using episomally located plasmids in a paratransgenic system is the persistence of these recombinant strains in the tsetse fly in the absence of antibiotic selection as premature loss of expression due to plasmid instability would not be desirable. Therefore, we assessed the stability of the recombinant strains during in vitro culture maintenance and after re-introduction in the tsetse fly. Results from the bacterial plate assay showed that in the absence of selection pressure in the culture medium the FliCpelBNb46fliC plasmid proved to be stably maintained with $94 \%$ of the 


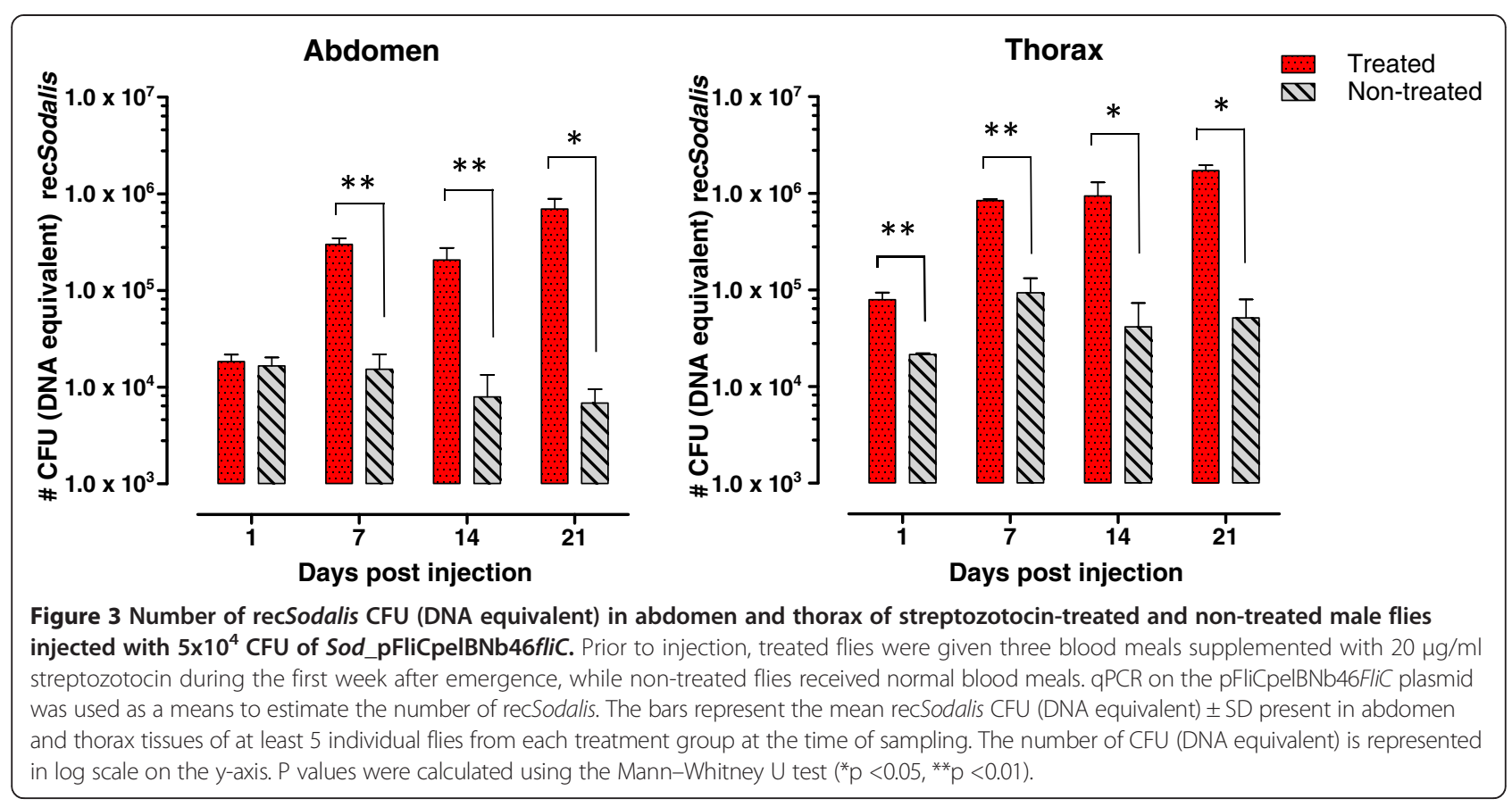

Sodalis population retaining the plasmid after 27 generations. This strong long-term plasmid retention in recSodalis was not always observed as for another strain (containing the FliCpelBNb33fliC plasmid) a complete plasmid loss in the total Sodalis population after 20 generations in culture. We postulate that the strong persistence of the FliCpelBNb46fliC plasmid in the recSodalis culture in the absence of a selection pressure is related to the high plasmid copy number that is observed in this strain and which was 20 fold higher than in the unstable Sod_FliCpelBNb33fliC strain.

Sod_FliCpelBNb46fliC remained present at high densities in the fly throughout the 28 day observation period. Furthermore, recSodalis was able to disseminate into the

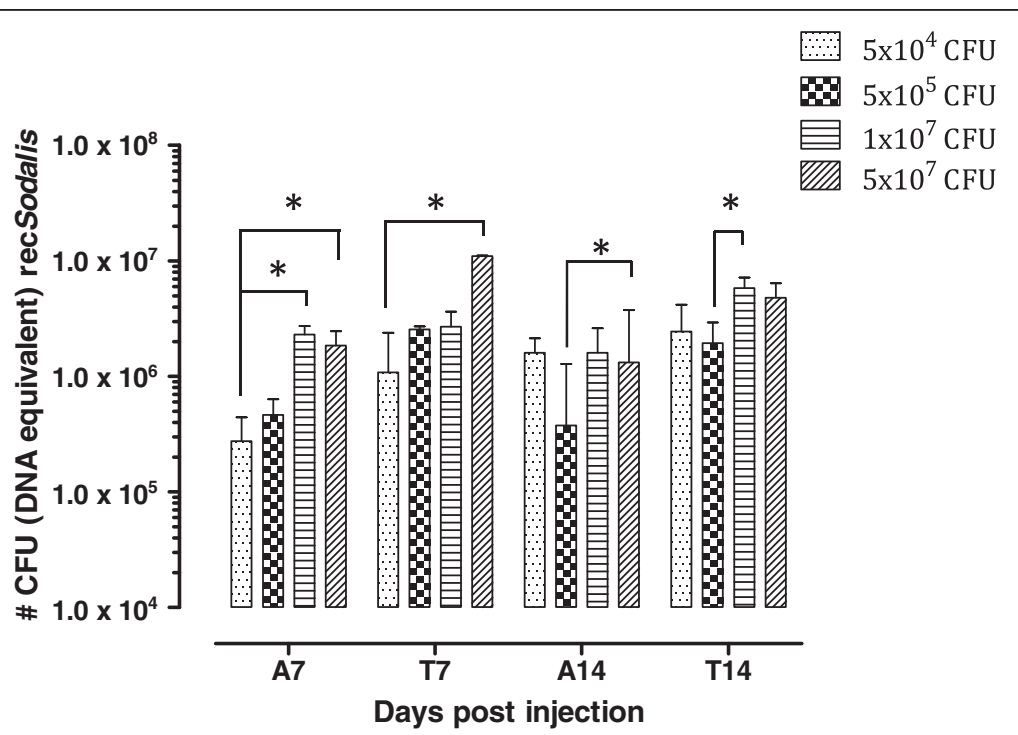

Figure 4 Number of recSodalis CFU (DNA equivalent) present in abdomen (A) and thorax (T) tissues of flies injected with respectively $5 \times 10^{4}, 5 \times 10^{5}, 1 \times 10^{7}$ and $5 \times 10^{7} \mathrm{CFU}$ of Sod_pFliCpelBNb46fliC. qPCR on the pFliCpelBNb46FliC plasmid was used as a means to estimate the number of recSodalis. The bars represent the mean recSodalis CFU (DNA equivalent) \pm SD present in abdomen and thorax tissues of at least 5 individual flies from each treatment group at the time of sampling. The number of CFU (DNA equivalent) is represented in log scale on the $y$-axis. P values were calculated using the Kruskal-Wallis test followed by Dunn's test for multiple comparison $\left({ }^{*} \mathrm{p}<0.05,{ }^{* *} \mathrm{p}<0.01\right)$. 


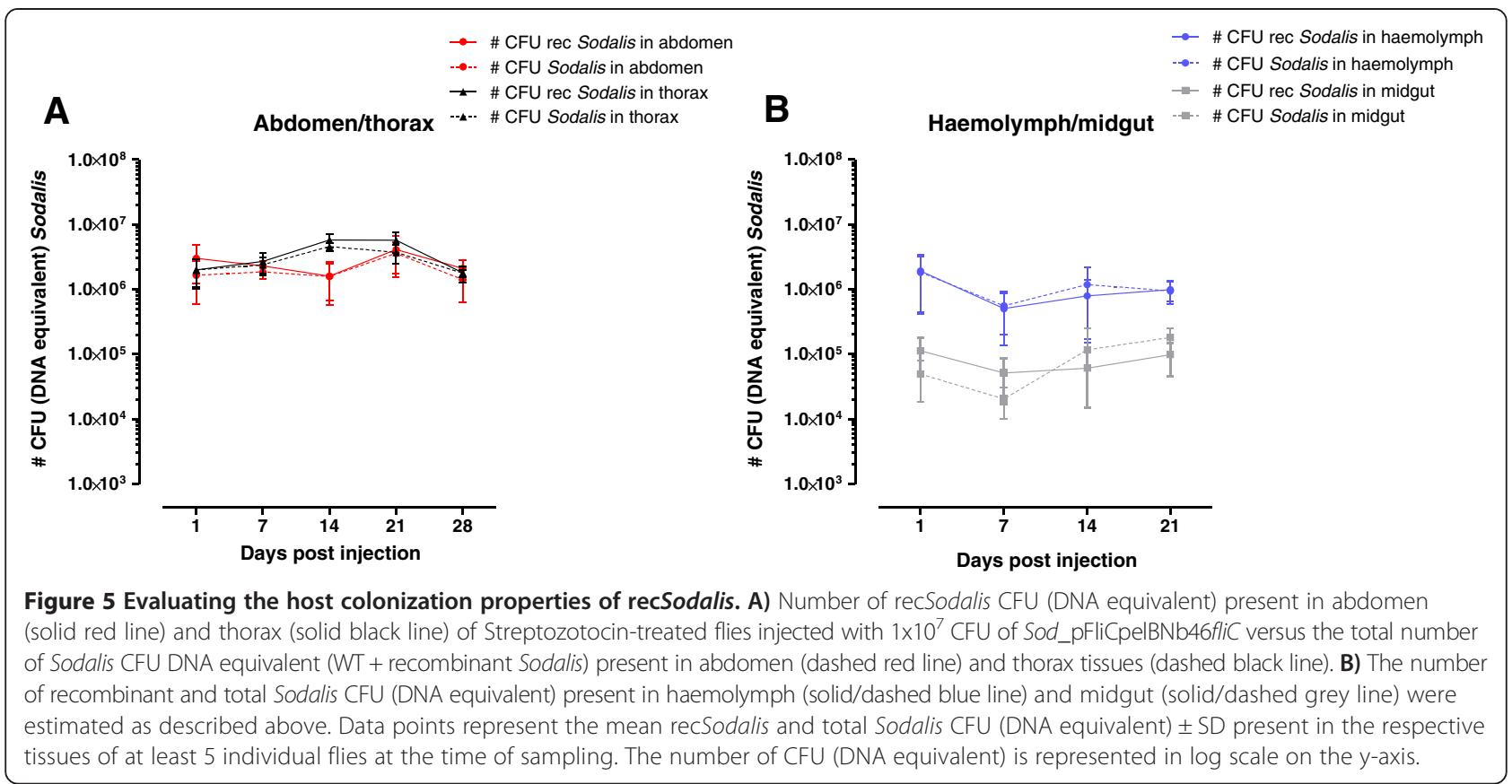

digestive tract reaching densities comparable to those in flies harboring WT symbionts. Furthermore, the majority of the total Sodalis population remained recombinant for the duration of the experiment indicating that the high plasmid stability that was observed in the in vitro culture is also present in the in vivo tsetse fly environment.

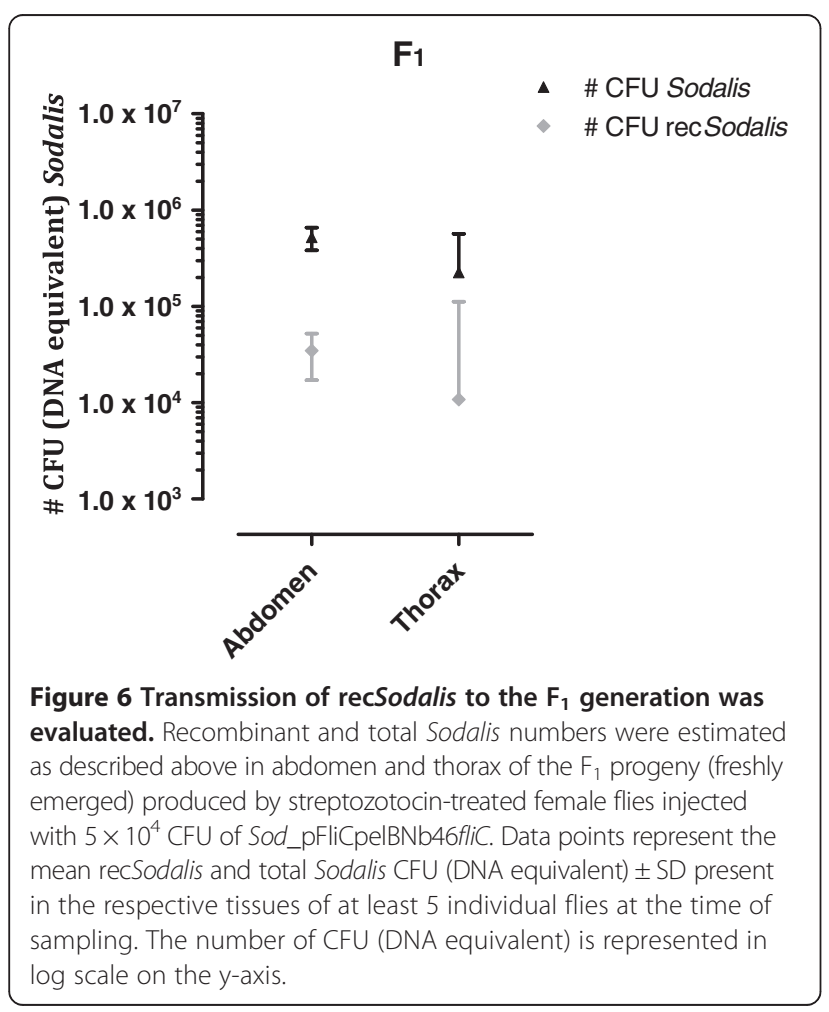

Although transmission to the $\mathrm{F}_{1}$ generation was observed, this seemed to be highly inefficient as only a small percentage of the total Sodalis population was found to be recombinant. This could be due to plasmid loss by recSodalis during the 30-day pupal stage or the inability of recSodalis to efficiently colonize the milk glands upon injection in the adult female fly which is a prerequisite for recSodalis transmission to the intrauterine larvae through the milk secretion. Indeed analysis of transcripts encoding Sodalis motility genes, fliC and $\operatorname{mot} A$, and cell invasion genes, $i n v A 1$ and $i n v A 2$, are up regulated in the larval and early pupal stages, and not in adult tsetse flies [15]. These results indicate that the Type-III secretion system and flagellum may be important for the transmission and establishment of symbiont infections in the intra-uterine progeny. Our results clearly indicate that for a successful use of Sodalis as paratransgenic vehicle in the tsetse fly several methodologies should still be improved allowing i) the generation of more stable recSodalis i.e. through an efficient methodology to insert exogenous DNA directly into the bacterial genome and ii) a more efficient transfer of the recSodalis to the next generations.

RecSodalis was found to continuously express functional nanobody in the tsetse fly as indicated by the $\mathrm{Nb}$ _An46 accumulation in the haemolymph over time. Abdomen and midgut extracts from the majority of insects that carried the recombinant nanobody-producing bacteria were positive in the ELISA, indicating the presence of functional $\mathrm{Nb}$ in these tissues. However the abundant presence of proteolytic enzymes in the tsetse fly 


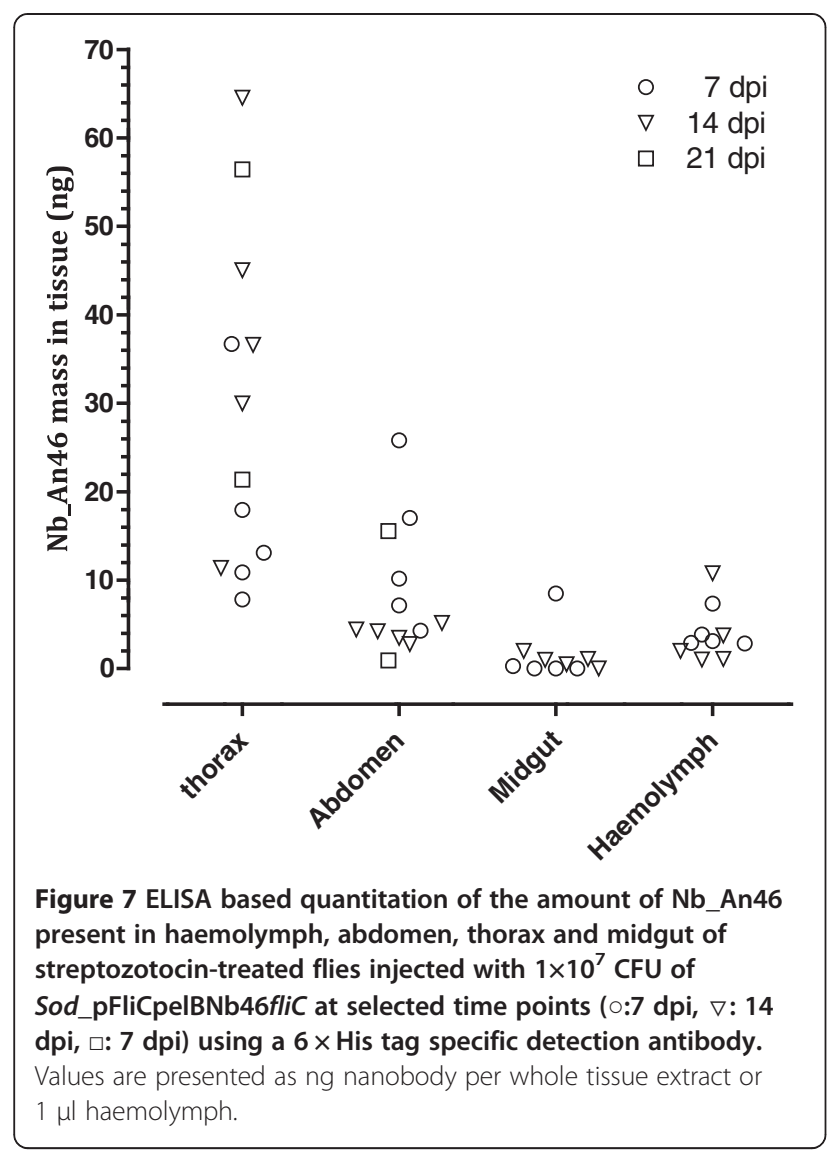

midgut probably interfered with detection, prohibiting accurate quantification of the $\mathrm{Nb}$ expression in this tissue. These results suggest the benefit of lowering the susceptibility of potential effector proteins to proteolytic degradation especially when they have to be functional in a digestive tissue such as the tsetse fly midgut. Nbs have the advantage that they can be mutagenized and selected for increased proteolytic stability [16].

From our experimental data, the expected levels of in vivo $\mathrm{Nb}$ expression are in the lower ng range. However, these levels of $\mathrm{Nb}$ expression should be sufficient to interfere with trypanosome development given that an infective blood meal of a tsetse fly in nature is estimated to contain around $10^{3}$ parasites as the average parasitaemia in T. brucei infected cattle fluctuates from $1.5 \times 10^{5}$ parasites per $\mathrm{ml}$ during the acute phase of infection to $5 \times 10^{4}$ parasites per $\mathrm{ml}$ during the chronic phase [17] and in vitro studies have shown that nanomolar concentrations of trypanolytic Nbs are sufficient to saturate $>95 \%$ of the surface VSG molecules and to provoke efficient trypanosome lysis [13,18]. Indeed, since a VSG:Nb molar ratio of 1:20 is sufficient to cause efficient lysis, $5 \mathrm{ng}$ of Nb_An46 should be adequate to efficiently lyse $10^{3}$ parasites.

\section{Conclusion}

These data are the first to show the potential of Sodalis as a delivery system for anti-trypanosome effector molecules in tsetse fly tissues relevant for trypanosome development. Given the ability of recombinant $S$. glossinidius to efficiently establish in different tsetse fly tissues at high densities and their capacity to release significant levels of functional anti-trypanosome Nbs in tissues were trypanosomes reside, the foundation has been laid for further exploration of the inhibitory effect on trypanosome development in the tsetse fly. Moreover, a paratransgenic approach using Sodalis to deliver Nbs that target the trypanosome-tsetse fly crosstalk could open a new avenue to unravel the molecular determinants of this specific parasite-vector association.

\section{Material and methods}

Insects, bacterial strains and culture conditions

G. morsitans morsitans (Westwood) from the colony at the Institute of Tropical Medicine Antwerp (ITM), originated from pupae collected in Kariba (Zimbabwe) and Handeni (Tanzania), were used in all experiments. Flies, maintained at $26^{\circ} \mathrm{C}$ and $65 \%$ relative humidity, were fed 3 days per week with defibrinated bovine blood using an artificial membrane system. Sodalis glossinidius strains used in this study were isolated from the haemolymph of surface-sterilized tsetse flies from the colony maintained at ITM. Cultures were maintained in vitro at $27^{\circ} \mathrm{C}$ in liquid Mitsuhashi-Maramorosch (MM) insect medium (PromoCell) supplemented with 10\% (v/v) heat-inactivated fetal bovine serum (FBS). Where appropriate, selection antibiotics were added to the media at the following concentrations: $100 \mu \mathrm{g} / \mathrm{ml}$ of ampicillin or $50 \mu \mathrm{g} / \mathrm{ml}$ of kanamycin. Flies used in this study were maintained at $26^{\circ} \mathrm{C}$ and $65 \%$ relative humidity, and fed 3 days per week with defibrinated bovine blood using an artificial membrane system.

\section{Plasmid constructs}

In this study the pFliCpelBNb46fliC plasmid was used in which a $6 \times$ His tagged $N b \_A n 46$ gene was fused to two secretion signals (FliC and pelB) and cloned into the multiple cloning site of the pCM66 expression vector. The pFliCpelBNb46fliC plasmid was derived from pFliCpelBNb33fliC [12] by replacement of pelBNb_An33 between the XbaI and EcoRI sites by pelBNb_An46 amplified as a XbaI-EcoRI fragment ( $465 \mathrm{bp})$ by PCR from the pHen6C plasmid containing the pelBNb_An46 gene using the following primer set: Nb46_FW, 5'-TCTAGAATGAAATACCTATTGCCTACGG-3' and Nb46_Rev, 5'GAATTCTTAGTGATGGTGATGGTGGTGGCGGCCG CGTGAGGAGAC-3' (XbaI-EcoRI restriction sites are underlined). 


\section{Transformation of Sodalis glossinidius}

Sodalis transformation with the pFliCpelBNb46fliC plasmid was conducted using a heat-shock based method as described in [14]. Following transformation, the cells were allowed to recover overnight at $27^{\circ} \mathrm{C}$ in liquid $\mathrm{MM}$ medium prior to plating.

\section{Growth curve measurements}

Logarithmically growing cultures were used to inoculate $25 \mathrm{ml}$ of MM-medium to an optical density at $600 \mathrm{~nm}$ $\left(\mathrm{OD}_{600}\right)$ of 0.05 . Sodalis cultures harboring the FliCpelBNb46fliC plasmid were allowed to grow without shaking for the first $24 \mathrm{~h}$, after which they were transferred to a shaking incubator. Samples were taken every $24 \mathrm{~h}$ for optical density measurements and $\mathrm{Nb}$ protein quantification in culture supernatants and whole cell extracts. The cell population doubling time was calculated from the growth rate during exponential growing phase using the following equation: doubling time (in hours) = $\mathrm{h} * \ln (2) / \ln (\mathrm{c} 2 / \mathrm{c} 1)$ where $\mathrm{c} 1$ is the initial concentration and $\mathrm{c} 2$ is the concentration when cultures reached maximum densities.

\section{Plasmid copy number assay}

The plasmid copy number per cell was calculated by dividing the absolute copy number by the number of Sodalis CFU present in the culture. The absolute number of plasmid and Sodalis CFU present in the Sod_FlicpelBNb46fliC culture grown in the presence of antibiotic selection (kanamycin $50 \mu \mathrm{g} / \mathrm{ml}$ ) was estimated by quantitative real-time PCR (qPCR) on DNA extracted from culture samples taken during the lag and exponential growth phases using the QIAGEN DNeasy extraction kit (QIAGEN). PCR reactions were carried out on a LightCycler $^{\mathrm{Tm}}$ (Roche Diagnostics, Mannheim, Germany). To estimate the number of Sodalis CFU present in the culture, a standard curve was generated using DNA extracts from a serial dilution series (1:10) ranging from $10^{7}$ Sod_FlicpelBNb46fliC CFU/ml to $10^{2}$ Sod_FlicpelBNb46fliC CFU/ml. For pFliCpelbNb46fliC plasmid number determination a dilution series (1:10) ranging from $10-10^{10}$ plasmid copies $\mu \mathrm{l}^{-1}$ was prepared to establish a linear standard curve for real-time PCR assays. For Sodalis, primers that target a 120-bp region of the single-copy $S$. glossinidius exochitinase gene were used: Qchi_Fw, 5'- TGGGGACAGTACGATGGCAG AGC -3 ; Qchi_Rev, 5'- TCATAGGCGGTCGGGGAT AATTGCG -3'. For the plasmid number determination, a 433-bp common region present on both pFliCpelBNb33fliC and pFliCpelBNb46fliC plasmids was amplified using the following primer set: pCM66_Fw, 5'- CTTGGCCCTCACTGACAG-3'; pCM66_rev, 5'- G CAGCCCTGGTTAAAAAC-3'. qPCR was performed in a $20-\mu$ l reaction mixture volume containing $10 \mu \mathrm{l}$ of
$2 \times \mathrm{iQ}^{\mathrm{TM}}$ SYBR green supermix, $0.3 \mu \mathrm{M}$ of each primer, template (DNA ) and RNase-free sterile water to a final volume of $20 \mu \mathrm{l}$.

\section{Measuring in vitro plasmid stability}

The in vitro stability of the pFliCpelBNb46fliC plasmid in Sodalis was measured by maintaining recombinant bacteria in log phase growth for 27 generations in MM without kanamycin selection. Samples were taken every two to four generations and plated on MM blood agar with and without kanamycin. The ratio of the number of colony forming units (CFU) grown on the selective blood agar plate to those grown on the non-selective blood agar was used to determine the percentage of plasmid-carrying cells. This ratio allows to monitor the plasmid stability during subsequent generations in the cultured recSodalis.

\section{Western blot analysis}

Cells were pelleted from bacterial cultures by centrifugation (15 min, $10000 \times \mathrm{g}$ ) and the supernatant was clarified from residual bacterial cells by a second centrifugation step. Proteins in the growth medium were precipitated with $10 \%$ trichloroacetic acid (TCA) for $1 \mathrm{~h}$ on ice. For SDS-PAGE, samples were heat denatured at $95^{\circ} \mathrm{C}$ in the presence of SDS-PAGE loading buffer containing $\beta$-mercaptoethanol and analyzed on a $12 \%(\mathrm{w} / \mathrm{v})$ polyacrylamide gel (Biorad). Proteins were transferred onto a Hybond $\mathrm{C}$ nitrocellulose membrane (Whattman). After overnight blocking with $1 \%$ $(\mathrm{w} / \mathrm{v})$ bovine serum albumin, the membrane was incubated sequentially with a mouse anti- $6 \times$ His-tag IgG1 antibody (1:1000) (Serotec) and a rabbit anti-mouse-IgG antibody (1:1000) (Serotec) conjugated to horseradish peroxidase. In between these successive $2 \mathrm{~h}$ incubations, the membrane was washed with PBS-0.1\% Tween 20. Thirty minutes after adding the substrate (TMB 1-Component Membrane Peroxidase Substrate, KPL) the reaction was stopped by washing the membrane with water.

\section{RecSodalis introduction into tsetse flies}

To evaluate the effect of reducing the WT Sodalis population in tsetse on recSodalis growth inside its host, newly emerged male G. morsitans morsitans flies were divided into two experimental groups. The treated group was given three blood meals supplemented with $20 \mu \mathrm{g} / \mathrm{ml}$ streptozotocin while the non-treated group received normal blood meals. Next, treated and non-treated flies were briefly anaesthetized by cold shock and microinjected intrathoracically with a suspension of $5 \times 10^{4}$ Sod_pFliCpelBNb46fliC CFU, using a $5 \mu$ l Hamilton 75RN microsyringe with gauge 33 removable electrotapered needles. After injection, males were kept in separate cages and received an antibiotics-free blood meal every $48 \mathrm{~h}$ throughout the course of the experiment. 
The optimal recSodalis injection dose was evaluated by injecting streptozotocin treated adult male flies with respectively $5 \times 10^{4}, 5 \times 10^{5}, 10^{7}$ and $5 \times 10^{7}$ CFU Sod_pFliCpelBNb46fliC followed by determination of the number of recSodalis CFU in abdomen and thorax 7 and 14 days post-injection.

To evaluate the in vivo persistence of Sod_pFliCpel$\mathrm{BNb} 46 f l i C$, streptozotocin treated adult male flies were injected intrathoracically with $10^{7} \mathrm{Sod}$ _pFliCpelBNb46fliC CFU. At different time points post-injection i.e. day 1 (teneral), 7, 14, 21 and 28, the number of WT and recSodalis CFU present in abdomen, thorax, haemolymph and midgut was measured using qPCR. To evaluate the transmission of recSodalis to the $F_{1}$ progeny, streptozotocin-treated female flies were injected intrathoracically with $5 \times 10^{4} \mathrm{Sod}$ :pFliCpelBNb46fliC CFU. Females were allowed to mate with WT males and their offspring (teneral stage) was evaluated for the presence of recSodalis.

\section{Measuring in vivo Sodalis and Wigglesworthia density}

At various time points post-injection flies were sacrificed for genomic DNA extraction using the QIAGEN DNeasy extraction kit (QIAGEN). We used a quantitative PCR (qPCR) method for the estimation of the number of WT Sodalis and recSodalis cells in tsetse fly tissues (abdomen, thorax, midgut and haemolymph). For this, triplicate cultures of WT and recSodalis were serially diluted (10-fold) in PBS to yield a Sodalis density ranging from $10^{7} \mathrm{CFU} / \mathrm{ml}$ to $10^{2} \mathrm{CFU} / \mathrm{ml}$. DNA was extracted from each Sodalis dilution followed by qPCR using primers that amplify a region of the pCM66 plasmid backbone present in recSodalis: (pCM66_Fw, 5'-CTTGGCCCTCACTGACAG-3' and pC M66_Rev, 5'- GCAGCCCTGGTTAAAAAC-3') and primers that target a 120-bp region of the single-copy Sodalis glossinidius exochitinase (Qchi) gene to determine the corresponding $\mathrm{Ct}$ values. Standard curves were generated by plotting these $C_{t}$ values against the corresponding $\log$ of Sodalis CFU/ml. This qPCR approach in combination with the Sodalis CFU standard curves allowed us to estimate the CFU (DNA equivalent) values present in the tsetse fly tissues of the different experimental fly series. An internal control was included to evaluate the DNA extraction efficiency in all the tissue samples. For this, samples were spiked with $0.4 \mathrm{ng}$ of plasmid DNA (pBAD24_GFP) prior to extraction. qPCR with $g f p$-specific primers (pBAD24_GFP: GFP_Fw, 5'-TGGCCAAC ACTTGTCACTAC-3' and GFP_Rev, 5'-AGAAGGACC ATGTGGTC-3') revealed a $C_{t}$ value of approximately 24.5 in all the DNA extracts corresponding to the $C_{t}$ value of the plasmid dilution used to spike to internal control alone demonstrating a uniform highly efficient extraction of the DNA in all the samples.

Wigglesworthia quantification was done by amplifying the thiamine locus with the following primer set: QthiC_F,
5'AAGTTATGATAGAAGGACCAGGAC-3' and QthiC R, 5'-CCCGGAGCAATATCAGTAGTTAG-3'. All Ct values were normalized to the G. morsitans morsitans reference gene $\beta$-actin for each sample using the following primer set: $\beta$-actin_F, 5'-GGCTTCTGGTCGTACTACT-3' and $\beta$-actin_R, 5'-CCGGACATCACAATGTTGG-3'. For each sample the obtained $\mathrm{Ct}$ value of the thiamine gene was normalized to the $C t$ value of the reference gene $(\beta$ actin) by subtracting the $\beta$-actin $\mathrm{Ct}$ value from the thiamine $\mathrm{Ct}$ value to yield a $\Delta \mathrm{Ct}$ value for each sample. $\Delta \mathrm{Ct}$ values were log-transformed and compared using the MannWhitney $U$ test to establish differences among treated and control groups. A value of $\mathrm{p}<0.05$ was considered significant in all analyses.

qPCR was performed in a $20-\mu$ l reaction mixture volume containing $10 \mu \mathrm{l}$ of $2 \times \mathrm{iQ}^{\mathrm{m} \mathrm{M}}$ SYBR green supermix, $0.3 \mu \mathrm{M}$ of each primer, template (genomic DNA, standard) and RNase-free sterile water to a final volume of $20 \mu \mathrm{l}$. The amount of fluorescence generated was measured during each amplification cycle using the following program: (i) initial denaturing at $95^{\circ} \mathrm{C}$ for $3 \mathrm{~min}$; (ii) 40 cycles, with 1 cycle consisting of denaturation at $95^{\circ} \mathrm{C}$ for $10 \mathrm{~s}$, annealing at $60^{\circ} \mathrm{C}$ for $10 \mathrm{~s}$, and extension at $72^{\circ} \mathrm{C}$ for $30 \mathrm{~s}$. All assays were carried out on a LightCycler ${ }^{\text {ms }}$ (Roche Diagnostics, Mannheim, Germany) in 96-well format plates in duplicate and were averaged for each sample. For each PCR run, a negative (no-template) control was used to test for false-positive results or contamination.

\section{Analysis of in vitro and in vivo Nanobody concentrations using ELISA}

i. In vitro. The amount of active $\mathrm{Nb}$ _An46 present in cytoplasmic extracts and growth medium was quantified using an optimized nanobody-detection ELISA [19]. For this purpose, Maxisorb 96-well plates (Nunc) were coated overnight $\left(4^{\circ} \mathrm{C}\right)$ with $200 \mathrm{ng}$ purified soluble AnTat 1.1 VSG per well in $0.1 \mathrm{M} \mathrm{NaHCO}_{3}, \mathrm{pH}$ 8.2. Residual protein binding sites were blocked for two hours at room temperature with $0.5 \%$ bovine serum albumin (BSA) in PBS. Standards and samples were added for $1 \mathrm{~h}$ at room temperature. Detection of antigen-bound nanobodies was performed with a mouse anti- $6 \times$ His IgG antibody (Serotec) directly conjugated to horseradish peroxidase. Thirty minutes after adding peroxidase substrate, the reaction was stopped with $0.33 \mathrm{M}$ $\mathrm{H}_{2} \mathrm{SO}_{4}$ and the optical density was measured at $450 \mathrm{~nm}$ (690 nm was used as reference filter). Protein concentrations were calculated from a standard curve fitted to a four parameter logistic equation using the Ascent software (Labsystems). Samples from the in vitro Sodalis culture were taken at the same time points indicated for the growth curve measurements. For each sample, $1 \mathrm{ml}$ of culture media was centrifuged 
two times $(8000 \times \mathrm{g})$ to obtain the extracellular and whole cell fractions. The whole cell extracts were prepared by resuspending the cell pellets in $0.2 \mathrm{ml}$ PBS supplemented with complete protease inhibitor (Roche) followed by sonication at an amplitude of 10 microns for 5 seconds (3 cycles on ice). For quantification of $\mathrm{Nb}$ _An46 present in cytoplasmic extracts and growth medium, a standard serial dilution series (1:2) starting from 2500 to $5 \mathrm{ng} / \mathrm{ml}$ of purified $\mathrm{Nb} \_$An46 was prepared in PBS and MM-medium respectively. MM medium and PBS alone were included as blanks.

ii. In vivo. Quantification of active $\mathrm{Nb}$ _An46 present in different tsetse fly tissues samples (abdomen, thorax, midgut and haemolymph) was performed at different time points post-injection. The preparation of the whole tissue extracts by sonication and the $\mathrm{Nb}$-quantification by ELISA were performed as described above. To mimic the sample complexity of the abdomen, thorax, midgut and haemolymph extracts, nanobody standards were prepared in the corresponding tissue extracts from non-injected wildtype flies. The respective tissues from flies harboring WT Sodalis were included as blanks.

\section{Additional file}

Additional file 1: Figure S1. The presence of Wigglesworthia in abdomen tissues from streptozotocin treated and non-treated male flies. Figure S2. The presence of Wigglesworthia in abdomen of male flies injected with 107 CFU of Sod_pFliCpelBNb46flic at day 7, 14 and 21 post injection and control flies.

\section{Competing interests}

The authors declare that they have no competing interests.

\section{Authors' contributions}

Conceived and designed the experiments: LDV, GC, JVDA. Performed the experiments: LDV, GC, KDR. Wrote the paper: LDV, GC, JVDA. All authors read and approved the final manuscript.

\section{Acknowledgments \\ This research was supported by an ITM SOFI grant, the InterUniversity Attraction Pole programme (IAP), FWO and the ERC-Starting Grant 'NANOSYM'. This work is also performed in the frame of a FAO/IAEA Coordinated Research Project on "Improving SIT for tsetse flies through research on their symbionts and pathogens". We wish to acknowledge the technical assistance of Jos Van Hees.}

\section{Author details}

'Department of Biomedical Sciences, Unit of Veterinary Protozoology, Institute of Tropical Medicine Antwerp (ITM), Antwerp, Belgium. ${ }^{2}$ Unit of Cellular and Molecular Immunology, Vrije Universiteit Brussel, Brussels, Belgium. ${ }^{3}$ Laboratory of Myeloid Cell Immunology, VIB, Brussels, Belgium. ${ }^{4}$ Department of Physiology, Laboratory of Zoophysiology, University of Ghent, B-9000 Ghent, Belgium.

Received: 27 August 2014 Accepted: 22 October 2014

Published online: 07 November 2014

\section{References}

1. Coutinho-Abreu IV, Zhu KY, Ramalho-Ortigao M: Transgenesis and paratransgenesis to control insect-borne diseases: current status and future challenges. Parasitol Int 2010, 59:1-8.
2. Durvasula RV, Gumbs A, Panackal A, Kruglov O, Aksoy S, Merrifield RB, Richards FF, Beard CB: Prevention of insect-borne disease: an approach using transgenic symbiotic bacteria. Proc Natl Acad Sci U S A 1997, 94:3274-3278

3. Fang W, Vega-Rodriguez J, Ghosh AK, Jacobs-Lorena M, Kang A, St Leger RJ: Development of transgenic fungi that kill human malaria parasites in mosquitoes. Science 2011, 331:1074-1077.

4. Wang S, Ghosh AK, Bongio N, Stebbings KA, Lampe DJ, Jacobs-Lorena M: Fighting malaria with engineered symbiotic bacteria from vector mosquitoes. Proc Natl Acad Sci U S A 2012, 109:12734-12739.

5. Attardo GM, Guz N, Strickler-Dinglasan P, Aksoy S: Molecular aspects of viviparous reproductive biology of the tsetse fly (Glossina morsitans morsitans): Regulation of yolk and milk gland protein synthesis. J Insect Physiol 2006, 52:1128-1136.

6. Cheng Q, Aksoy S: Tissue tropism, transmission and expression of foreign genes in vivo in midgut symbionts of tsetse flies. Insect Mol Biol 1999, 8:125-132.

7. Welburn SC, Maudlin I, Ellis DS: In vitro cultivation of rickettsia-like-organisms from Glossina spp. Ann Trop Med Parasitol 1987, 81:331-335.

8. Beard CB, O'Neill SL, Mason P, Mandelco L, Woese CR, Tesh RB, Richards FF, Aksoy S: Genetic transformation and phylogeny of bacterial symbionts from tsetse. Insect Mol Biol 1993, 1:123-131.

9. Attardo GM, Lohs C, Heddi A, Alam UH, Yildirim S, Aksoy S: Analysis of milk gland structure and function in Glossina morsitans: milk protein production, symbiont populations and fecundity. J Insect Physiol 2008, 54:1-7.

10. Van Den Abbeele J, Bourtzis K, Weiss B, Cordón-Rosales C, Miller W, Abd-Alla AM, Parker A: Enhancing tsetse fly refractoriness to trypanosome infection-a new IAEA coordinated research project. J Invertebr Pathol 2013, 112:S142-S147.

11. Caljon G, De Vooght L, Van Den Abbeele J: Options for the delivery of Nanobodies as anti-pathogen molecules in arthropod vectors. J Invertebr Pathol 2013, 112(Suppl):S75-S82.

12. Hamers-Casterman C, Atarhouch T, Muyldermans S, Robinson G, Hamers C, Songa EB, Bendahman N, Hamers R: Naturally occurring antibodies devoid of light chains. Nature 1993, 363:446-448.

13. Stijlemans B, Caljon G, Natesan SK, Saerens D, Conrath K, Perez-Morga D, Skepper JN, Nikolaou A, Brys L, Pays E, Magez S, Field MC, De Baetselier P, Muyldermans S: High affinity nanobodies against the Trypanosome brucei VSG are potent trypanolytic agents that block endocytosis. PLOS Pathog 2011, 7:e1002072.

14. De Vooght L, Caljon G, Stijlemans B, De Beatselier P, Coosemans M, Van Den Abbeele J: Expression and extracellular release of a functional anti-trypanosome Nanobody ${ }^{\circledR}$ in Sodalis glossinidius, a bacterial symbiont of the tsetse fly. Microb Cell Fact 2012, 11:23.

15. Toh H, Weiss BL, Perkin SA, Yamashita A, Oshima K, Hattori M, Aksoy S: Massive genome erosion and functional adaptations provide insights into the symbiotic lifestyle of Sodalis glossinidius in the tsetse host. Genome Res 2006, 16:149-156.

16. Harmsen MM, van Solt CB, van Zijderveld-van Bemmel AM, Niewold TA, van Zijderveld FG: Selection and optimization of proteolytically stable llama single-domain antibody fragments for oral immunotherapy. App/ Microbiol Biotechnol 2006, 72:544-551.

17. Van den Bossche P, Ky-Zerbo A, Brandt J, Marcotty T, Geerts S, De DR: Transmissibility of Trypanosoma brucei during its development in cattle. Trop Med Int Health 2005, 10:833-839.

18. Caljon G, Stijlemans B, Saerens D, Van Den Abbeele J, Muyldermans S, Magez S, De Baetselier P: Affinity is an important determinant of the anti-trypanosome activity of nanobodies. PLoS Negl Trop Dis 2012, 6:e1902.

19. Caljon G, Caveliers V, Lahoutte T, Stijlemans B, Ghassabeh GH, Van den Abbeele J, Smolders I, De Baetselier P, Michotte Y, Muyldermans S, Magez S, Clinckers R: Using microdialysis to analyse the passage of monovalent nanobodies through the blood-brain barrier. Br J Pharmacol 2012, 165:2341-2353.

doi:10.1186/s12934-014-0156-6

Cite this article as: De Vooght et al:: Delivery of a functional anti-trypanosome Nanobody in different tsetse fly tissues via a bacterial symbiont, Sodalis glossinidius. Microbial Cell Factories 2014 13:156 